\title{
Q-SPECIAL FUNCTIONS AND THEIR OCCURRENCE IN QUANTUM GROUPS
}

\author{
T. H. KOORNWINDER
}

\begin{abstract}
SUMMARY
In classical Lie group theory irreducible representation spaces are often decomposed into subspaces belonging to irreducible representations of a subgroup (or a chain of subgroups). Matrix elements of the representation w.r.t. a basis obtained in this way can often be expressed in terms of special functions. In particular, spherical functions are the matrix elements being left and right invariant w.r.t. a subgroup forming a Gelfand pair with the big group. An elementary example is the group $S U(2)$ with diagonal subgroup isomorphic to $U(1)$. Then the matrix elements of the irreducible representations of $S U(2)$ w.r.t. a $U(1)$-basis can be expressed in terms of Jacobi polynomials and the spherical functions are Legendre polynomials. Nothing essential changes when we take the basis w.r.t. a subgroup conjugate to $U(1)$, for instance $S O(2)$.

For quantum groups the situation is different. Only few quantum subgroups are available, in general, and we cannot take conjugates of quantum subgroups. For instance, quantum $S U(2)$ has a quantum subgroup $U(1)$ (an ordinary group), but no other nontrivial quantum subgroups are known and no conjugates of $U(1)$ can be taken. The matrix elements of the irreducible representations of quantum $S U(2)$ have been computed (cf. [5], [4], [1]) as little $q$-Jacobi polynomials and the spherical matrix elements as little $q$-Legendre polynomials. (See Koelink \& Koornwinder's $q$-special functions tutorial in these Proceedings.)

In order to say something about the missing conjugates of a quantum subgroup, we use the quantized universal enveloping algebra. Let $\mathcal{U}=\mathcal{U}_{q}(s l(2))$ be the algebra generated by $A, A^{-1}, B, C$ with relations $A A^{-1}=1=A^{-1} A$, $A B=q B A, A C=q C A,[B, C]=\left(A^{2}-A^{-2}\right) /\left(q-q^{-1}\right)$. U becomes a Hopf
\end{abstract}

1991 Mathematics Subject Classification. Primary 33D45, 33D80, $17 \mathrm{~B} 37$.

This paper is in final form and no version of it will be submitted for publication elsewhere.

(C) 1992 American Mathematical Society $0271-4132 / 92 \$ 1.00+\$ .25$ per page 
algebra with comultiplication $\Delta(A)=A \otimes A, \Delta(B)=A \otimes B+B \otimes A^{-1}, \Delta(C)=$ $A \otimes C+C \otimes A^{-1}$. Call $X \in \mathcal{U}$ twisted primitive if $\Delta(X)=A \otimes X+X \otimes A^{-1}$. Then $X$ has this property iff $X$ is a linear combination of $A-A^{-1}, B$ and $C$. This three-dimensional space is a kind of quantum analogue of the Lie algebra $\operatorname{sl}(2)$.

The Hopf *-algebra $\mathcal{A}$ of "polynomial functions" on quantum $S U(2)$ is in doubly non-degenerate Hopf algebra duality with $\mathcal{U}$. Call $a \in \mathcal{A}$ left (right) invariant w.r.t. a twisted primitive element $X \in \mathcal{U}$ if $(X \otimes$ id $)(\Delta(a))=0$ (respectively (id $\otimes X)(\Delta(a))=0)$.

Theorem ([2], [3]). Let $X_{1}$ and $X_{2}$ be twisted primitive elements in $\mathcal{U}$. Then the elements of $\mathcal{A}$ being left invariant w.r.t. $X_{1}$ and right invariant w.r.t. $X_{2}$ form a subalgebra generated by a single element $\rho$ of $\mathcal{A}$ which is quadratic in the generators of $\mathcal{A}$. The intersection of this subalgebra with the subspace of $\mathcal{A}$ belonging to an irreducible representation of quantum $S U(2)$ of odd dimension $2 l+1$ is one-dimensional and spanned by a certain Askey-Wilson polynomial of degree $l$ and argument $\rho$.

\section{REFERENCES}

1. T. H. Koornwinder, Representations of the twisted $S U(2)$ quantum group and some $q$ hypergeometric orthogonal polynomials, Nederl. Akad. Wetensch. Proc. Ser. A 92 (1989), 97-117.

2. - Orthogonal polynomials in connection with quantum groups, Orthogonal polynomials: Theory and Practice, P. Nevai (ed.), NATO ASI Series C, Vol. 294, Kluwer Academic Publishers, 1990, pp. 257-292.

3. - Askey-Wilson polynomials as zonal spherical functions on the $S U(2)$ quantum group, preprint, CWI Rep. AM-R9013, Amsterdam.

4. T. Masuda, K. Mimachi, Y. Nakagami, M. Noumi and K. Ueno, Representations of the quantum group $S U_{q}(2)$ and the little $q$-Jacobi polynomials, J. Funct. Anal. 99 (1991), 127-151.

5. L. L. Vaksman and Ya. S. SoǏbel'man, Algebra of functions on the quantum group SU(2), Functional Anal. Appl. 22 (1988), 170-181.

CWI, P.O. Box 4079, 1009 AB Amsterdam, The Netherlands 